\title{
SYMMETRY PROPERTIES OF A NONLINEAR ACOUSTICS MODEL
}

\author{
J C NDOGMO
}

\begin{abstract}
We give a classification into conjugacy classes of subalgebras of the symmetry algebra generated by the Zabolotskaya-Khokhlov equation, and obtain all similarity reductions of this equation into $(1+1)$-dimensional equations. We thus show that Lie classical reduction approach may also give rise to more general reduced equations as those expected from the direct method of Clarkson and Kruskal. By transforming the determining system for the similarity variables into the equivalent adjoint system of total differential equations, similarity reductions to ODEs which are independent of the three arbitrary functions defining the symmetries are also obtained. These results are again compared with those obtained by the direct method of Clarkson and Kruskal, by finding in particular equivalence transformations mapping some of the reduced equations to each other. Various families of new exact solutions are also derived.
\end{abstract}

\section{INTRODUCTION}

The Zabolotskaya-Khokhlov (ZK) equation is a nonlinear model of sound wave propagation derived from the incompressible Navier-Stokes equation 1, 2, 3. The $(2+1)$ - dimensional version of this equation has the form

$$
\Delta(t, x, y, u) \equiv u_{x t}-\left(u u_{x}\right)_{x}-u_{y y}=0,
$$

and it has been studied from the Lie group approach in a number of papers [4, 5, 6, 7]. Chowdhury and Naser [4] attempted the determination of the symmetry algebra of this equation and calculated some of their conservation laws. However, Schwarz [5] and Hereman [6] were certainly the first to provide independently the correct generators of the Lie symmetry algebra for this popular acoustics model. Although the calculation of symmetry algebras for almost all systems of differential equations has long been reduced to a mere function on a number of modern computing systems, many symmetry properties of this equation are still to be uncovered.

In [7, some similarity reductions of Equation (11) to $(1+1)$-dimensional models were obtained, based on the direct method of Clarkson and Kruskal 8. More specifically, it was shown that if a similarity solution of Equation (1) of the form

$$
u=U(t, x, y, W(\xi, \eta)), \quad \xi=\xi(t, x, y), \quad \eta=\eta(t, x, y)
$$

can be found by solving a reduced $(1+1)$-dimensional equation, then, when $\xi_{x} \neq 0$, such an equation must be one of three non-equivalent $(1+1)$-dimensional equations found in that paper. However, these reduced equations are either determined only

2000 Mathematics Subject Classification. 70G65, 83C15, 34C20.

Key words and phrases. Lie algebra classification, Comparison of reduction techniques, Equivalence transformations, Exact solutions. 
implicitly in terms of solutions of certain partial differential equations (PDEs), or they depend on up to four arbitrary functions, and the same can be said about their solutions. In the same paper, using again the direct method of Clarkson and Kruskal and a restrictive anzatz, the most general oDE that every similarity solution of (11) obtained by solving an ODE must satisfy is shown to be of the form

$$
w^{\prime 2}+w w^{\prime \prime}+(A z+B) w^{\prime}+2 A=\frac{1}{3}(A z+B)^{2} .
$$

Some of the unanswered questions raised in [7] were how to find all the non equivalent similarity reductions of the ZK equation to an ODE by the classical Lie symmetry approach, and whether there is any connection between these two types of reduction techniques. The first of these two questions stems from the fact that the symmetries of Equation (11) depend on three arbitrary functions of time, and so its similarity reductions are usually achieved by restricting these functions to some specific types of elementary functions, such as exponential or simple polynomial functions of time [4, 5. It also stems from the fact that no classification of low-dimensionial subalgebras of the ZK symmetry algebra into conjugacy classes is available.

In this paper, we obtain all canonical forms of non-equivalent one- and twodimensional subalgebras of the symmetry algebra $L$ of (1), under the adjoint representation of the symmetry group. We then apply them to obtain all similarity reductions of the ZK equation to $(1+1)$-dimensional equations, using the classical Lie approach. The same reductions are also obtained by direct case analysis. We thus show that in addition to its simpler algorithm and other properties, Lie classical reduction method gives rise not only to simpler equations, but it may also yield more unified and general reduced equations than those expected from the direct method (of Clarkson Kruskal). Next, by transforming the determining system for the similarity variables into the equivalent adjoint system of total differential equations, similarity reductions to large classes of ODEs which are independent of the three arbitrary functions defining the symmetries are also obtained. The latter system of total differential equations allows for an easier determination of the invariant functions defining the similarity coordinates. Large classes of similarity solutions depending on much less arbitrary functions than those obtained in [7] are also derived in this way.

Finally, we find equivalence transformations mapping some of the equations that we've obtained by the Lie classical method to some sub-equations of the reduced equation (3) obtained by the direct method of Clarkson and Kruskal. Our discussions also show that in principle any reduced equation achievable with the direct method can also be achieved by Lie classical method although the converse is totally out of question, as far as the properties of the reduced equations are concerned.

This paper is organized as follows. In the next section we discuss the symmetry algebra of the ZK equation and determine its algebraic structure as well as its connection with Kac-Moody-Virasoro (KMV) algebras. Section 3 is devoted to the classification of low-dimensional subalgebras of $L$ and Section 4 to the similarity reductions of the ZK equation. We investigate the connection between the two types of reductions invoked above in Section 5 . Some concluding remarks are given in the last section. 


\section{Symmetry group of the ZK EQUATION}

2.1. Structure of the symmetry algebra. The Lie algebra of the ZK equation is well known [5, 6]. This is the Lie algebra defined by the infinitesimal generators of the point symmetry group $G$ of the equation, that is, the Lie group of point transformations that map every solution of the equation to another solution of the same equation [9, 10]. These infinitesimal generators are vector fields of the form

$$
\mathbf{v}=\xi_{1}(t, x, y, u) \partial_{t}+\xi_{2}(t, x, y, u) \partial_{x}+\xi_{3}(t, x, y, u) \partial_{y}+\phi(t, x, y, u) \partial_{u}
$$

acting on the space of independent variables coordinatized by $(t, x, y)$ and the space of the dependent variable coordinatized by $u$, and such that the second prolongation $\operatorname{Pr}^{(2)} \mathbf{v}$ of $\mathbf{v}$ satisfies

$$
\left.\operatorname{Pr}^{(2)} \mathbf{v} \Delta(t, x, y, u)\right|_{\Delta(t, x, y, u)=0}=0
$$

Equation (5) completely determines the unknown functions $\xi_{1}, \xi_{2}, \xi_{3}, \phi$ defining $\mathbf{v}$ [9, 22, 10]. For the ZK equation, the linearly independent vector fields, i.e. the generators of the Lie symmetry algebra are given by the operators

$$
\begin{aligned}
\mathbf{v}_{0} & =2 x \partial_{x}+y \partial_{y}+2 u \partial_{u} \\
\mathbf{x}_{g} & =g \partial_{x}-g^{\prime} \partial_{u} \\
\mathbf{y}_{h} & =\frac{1}{2} y h^{\prime} \partial_{x}+h \partial_{y}-\frac{1}{2} y h^{\prime \prime} \partial_{u} \\
\mathbf{z}_{f} & =f \partial_{t}+\frac{1}{6}\left(2 x f^{\prime}+y^{2} f^{\prime \prime}\right) \partial_{x}+\frac{2 y}{3} f^{\prime} \partial_{y}+\frac{1}{6}\left(-4 u f^{\prime}-2 x f^{\prime \prime}-y^{2} f^{\prime \prime \prime}\right) \partial_{u}
\end{aligned}
$$

where $f, g, h$ are arbitrary $C^{\infty}$ functions of the time variable $t$ defined on some open subset of $\mathbb{R}$, assumed to be the based field of $L$, and where a prime represents a derivative with respect to $t$. The ZK symmetry algebra is therefore infinite-dimensional, and its commutation relations are easily found to be as follows

$$
\begin{aligned}
{\left[\mathbf{v}_{0}, \mathbf{x}_{g}\right] } & =-2 \mathbf{x}_{g}, & {\left[\mathbf{x}_{g}, \mathbf{y}_{h}\right] } & =0 \\
{\left[\mathbf{v}_{0}, \mathbf{y}_{h}\right] } & =-\mathbf{y}_{h}, & {\left[\mathbf{x}_{g}, \mathbf{z}_{f}\right] } & =\mathbf{x}_{\left(f^{\prime} g / 3-f g^{\prime}\right)} \\
{\left[\mathbf{v}_{0}, \mathbf{z}_{f}\right] } & =0, & {\left[\mathbf{y}_{h}, \mathbf{z}_{f}\right] } & =\mathbf{y}_{\left(\frac{2}{3} f^{\prime} h-f h^{\prime}\right)} \\
{\left[\mathbf{x}_{g_{1}}, \mathbf{x}_{g_{2}}\right] } & =0 & {\left[\mathbf{y}_{h_{1}}, \mathbf{y}_{h_{2}}\right] } & =\mathbf{x}_{\left(h_{1} h_{2}^{\prime}-h_{1}^{\prime} h_{2}\right) / 2} \\
{\left[\mathbf{z}_{f_{1}}, \mathbf{z}_{f_{2}}\right] } & =\mathbf{z}_{\left(f_{1} f_{2}^{\prime}-f_{1}^{\prime} f_{2}\right) .} & &
\end{aligned}
$$

These commutation relations show that the ZK symmetry algebra $L$ has the structure of a semi-direct sum Lie algebra, $L=\mathcal{R}+\mathcal{S}$, where $\mathcal{R}=\left\langle\mathbf{v}_{0}, \mathbf{x}_{g}, \mathbf{y}_{h}\right\rangle$ is the radical and $\mathcal{S}=\left\langle\mathbf{z}_{f}\right\rangle$ is the semisimple part, also called Levi factor of $L$. It is indeed a well-known fact [11] that the infinite dimensional Lie algebra generated by $\mathbf{z}_{f}$ is a simple Lie algebra, i.e. it has no nontrivial ideal. This is easily established by first nothing that by a result of Cartan [12, the Lie algebra $J(I)=\left\{f(t) \partial_{t}: f \in C^{\infty}(I)\right\}$ of vector fields on the open interval $I$ of $\mathbb{R}$ is a simple algebra, and that the map

$$
\sigma: J(I) \rightarrow \mathcal{S}: f(t) \partial_{t} \mapsto \mathbf{z}_{f(t)}
$$

is a Lie algebra isomorphism. We also note that the radical $\mathcal{R}$, which is solvable by definition, is actually nonnilpotent. Its nilradical, i.e. its maximal nilpotent ideal is generated by $\left\langle\mathbf{x}_{g}, \mathbf{y}_{h}\right\rangle$.

The commutation relations (7) also show that the infinite-dimensional subalgebra $L_{0}=\left\langle\mathbf{x}_{g}, \mathbf{y}_{h}, \mathbf{z}_{f}\right\rangle$ of $L$ can be identified with a subalgebra of a KMV type algebra. 
Indeed, by restricting the arbitrary functions $f, g$, and $h$ to be Laurent polynomials, the resulting commutation relations take the form

$$
\begin{array}{rlrl}
{\left[\mathbf{x}_{t^{m}}, \mathbf{x}_{t^{n}}\right]} & =0, & {\left[\mathbf{x}_{t^{m}}, \mathbf{y}_{t^{n}}\right]=0} \\
{\left[\mathbf{z}_{t^{m}}, \mathbf{z}_{t^{n}}\right]=(n-m) \mathbf{z}_{t^{m+n-1}},} & {\left[\mathbf{x}_{t^{m}}, \mathbf{z}_{t^{n}}\right]=\frac{m-3 n}{3} \mathbf{x}_{t^{m+n-1}}} \\
{\left[\mathbf{y}_{t^{m}}, \mathbf{z}_{t^{n}}\right]=\frac{2 m-3 n}{3} \mathbf{y}_{t^{m}+n-1},} & {\left[\mathbf{y}_{t^{m}}, \mathbf{y}_{t^{n}}\right]=\frac{n-m}{2} \mathbf{x}_{t^{m+n-1}},}
\end{array}
$$

and this shows that $\left\langle\mathbf{x}_{g}, \mathbf{y}_{h}\right\rangle$ generates the corresponding Kac-Moody subalgebra of $L_{0}$, while $\left\langle\mathbf{z}_{f}\right\rangle$ generates the Virasoro subalgebra [13]. These algebraic properties of the ZK equation, which can be linearized by a generalized hodograph transformation 18, are in agreement with a widespread belief about integrable $(2+1)$-dimensional equations. Indeed, the symmetry algebra of most integrable $(2+1)$-dimensional equations are infinite-dimensional and can be identified with a subalgebra of a KMV type algebra [11, 13, 14. There are nonetheless some exceptions provided for instance by the breaking soliton equation and the ZakharovStrachan equation [15] which are integrable but do not admit a KMV type symmetry algebra. On the other hand, the symmetry algebra of every known non-integrable equations has no KMV structure. This suggests that KMV structures are in some way which is still to be clarified associated with integrability. By an integrable equation here, we refer to equations allowing a Lax pair, an infinity of conservation laws, soliton and multisoliton solutions, a family of analytic periodic and quasi-periodic solutions, as well as a number of similar properties.

2.2. Group transformations. One of the most important properties of the symmetry group of a differential equation is to transform one solution of the equation into another solution of the same differential equation. These group transformations are generated by some one-parameter group of transformations, each of which is the local flow $\psi(\epsilon, \mathbf{w})$, where $\mathbf{w}=(t, x, y, u)$ and $\epsilon \in \mathbb{R}$, of a basis element $\mathbf{v}$ of the Lie symmetry algebra, acting on the space of independent and dependent variables. More specifically, the local flow $\psi(\epsilon, \mathbf{w})$ of $\mathbf{v}$ is given for every $\epsilon$ for which it is defined by

$$
\partial_{\epsilon} \psi(\epsilon, \mathbf{w}) \equiv \dot{\psi}(\epsilon, \mathbf{w})=\left.\mathbf{v}\right|_{\psi(\epsilon, \mathbf{w})}, \quad \text { and } \quad \psi(0, \mathbf{w})=\mathbf{w},
$$

where $\partial_{\epsilon}=d / d \epsilon$. The full group action can be obtained by combining all the one-parameter group actions determined by each infinitesimal generator. We will use the notation

$$
\psi(\epsilon, \mathbf{w})=\tilde{\mathbf{w}}=(\tilde{t}, \tilde{x}, \tilde{y}, \tilde{u}),
$$

and we let the generic generator of the ZK symmetry algebra have the form

$$
\mathbf{v}=k_{0} \mathbf{v}_{0}+\mathbf{x}_{g}+\mathbf{y}_{h}+\mathbf{z}_{f},
$$

where $k_{0}$ is a scalar. We also denote by $F^{\prime}$ the derivative of a function $F=F(t)$ of the time variable $t$. To find the flow of $\mathbf{v}$ for the ZK equation, we have to distinguish a number of cases.

Case 1: $g=h=f=0$, and $k_{0} \neq 0$

In this case $\mathbf{v}=\mathbf{v}_{0}$, and by assuming without loss of generality that $k_{0}=1$, we 
have

$$
\begin{aligned}
\partial_{\epsilon} \tilde{t} & =0 & & \partial_{\epsilon} \tilde{y}=\tilde{y} \\
\partial_{\epsilon} \tilde{x} & =2 \tilde{x} & & \partial_{\epsilon} \tilde{u}=\tilde{u} .
\end{aligned}
$$

Consequently, the corresponding group action is given by

$$
\psi(\epsilon, \mathbf{w})=\left(t, x e^{2 \epsilon}, y e^{\epsilon}, u e^{2 \epsilon}\right)=\tilde{\mathbf{w}} .
$$

It then follows that

$$
\tilde{u}(t, x, y)=e^{2 \epsilon} u\left(t, x e^{-2 \epsilon}, y e^{-\epsilon}\right)
$$

is a solution of the ZK equation whenever $u(t, x, y)$ is a solution.

Case 2: $f=h=0, \quad g \neq 0, k_{0}$ is arbitrary

We have in this case $\mathbf{v}=k_{0} \mathbf{v}_{0}+\mathbf{x}_{g}$.

We find that

Case 2a $: \quad k_{0}=0$

$$
\tilde{\mathbf{w}}=\left(t, x+\epsilon g, y, u-\epsilon g^{\prime}\right) .
$$

Consequently,

$$
\tilde{u}(t, x, y)=u(t, x-\epsilon g, y)-\epsilon g^{\prime}
$$

is a solution whenever $u(t, x, y)$ is.

Case 2b : $\quad k_{0} \neq 0$

In this case, $\tilde{\mathbf{w}}$ is given by

$$
\begin{aligned}
& \tilde{t}=t \\
& \tilde{x}=\frac{1}{2 k_{0}}\left(e^{2 k_{0} \epsilon}\left(2 k_{0} x+g\right)-g\right) \\
& \tilde{y}=y e^{k_{0} \epsilon} \\
& \tilde{u}=\frac{1}{2 k_{0}}\left(e^{2 k_{0} \epsilon}\left(2 k_{0} u-g^{\prime}\right)+g^{\prime}\right) .
\end{aligned}
$$

Therefore, if $u(t, x, y)$ is a solution, then

$$
\tilde{u}(t, x, y)=\frac{1}{2 k_{0}}\left[e^{2 k_{0} \epsilon}\left(2 k_{0} u^{*}-g^{\prime}\right)+g^{\prime}\right]
$$

is also a solution, where

$$
u^{*}=u\left[t, \frac{1}{2 k_{0}}\left(\left(2 k_{0} x+g\right) e^{-2 k_{0} \epsilon}-g\right), y e^{-k_{0} \epsilon}\right] .
$$

$\underline{\text { Case 3 }}: \quad f=0, h \neq 0$ and $g$ and $k_{0}$ are arbitrary We have two subcases in this case.

We have

$$
\text { Case 3a }: \quad k_{0}=0
$$

$$
\tilde{\mathbf{w}}=\left(t, x+g \epsilon+\epsilon(y+h \epsilon) h^{\prime}, y+2 h \epsilon, u-\epsilon\left(g^{\prime}+(y+h \epsilon) h^{\prime \prime}\right)\right) .
$$


Consequently, whenever $u(t, x, y)$ is a solution, so is

$$
\tilde{u}(t, x, y)=u^{*}-\epsilon\left(g^{\prime}+y(-h \epsilon) h^{\prime \prime}\right)
$$

where

$$
u^{*}=u\left(t, x-g \epsilon+\epsilon(-y+h \epsilon) h^{\prime}, y-2 h \epsilon\right) .
$$

$\underline{\text { Case } 3 \mathrm{~b}}: \quad k_{0} \neq 0$

We find that $\tilde{\mathbf{w}}$ is given by

$\tilde{t}=t$

$\tilde{x}=\frac{1}{2 k_{0}^{2}}\left[-k_{0} g+2 h h^{\prime \prime}+e^{k_{0} \epsilon}\left(-4 h h^{\prime}-2 k_{0} y h^{\prime}\right)+e^{2 k_{0} \epsilon}\left(k_{0} g+2 k_{0}^{2} x+2 h h^{\prime}+2 k_{0} y h^{\prime}\right)\right]$

$\tilde{y}=\frac{1}{k_{0}}\left[-2 h+e^{k_{0} \epsilon}\left(2 h+k_{0} y\right)\right]$

$\tilde{u}=\frac{-1}{2 k_{0}^{2}}\left[-k_{0} g^{\prime}+2 h h^{\prime \prime}+e^{k_{0} \epsilon}\left(-4 h h^{\prime \prime}-2 k_{0} y h^{\prime \prime}\right)+e^{2 k_{0} \epsilon}\left(-2 k_{0}^{2} u+k_{0} g^{\prime}+2 h h^{\prime \prime}+2 k_{0} y h^{\prime \prime}\right)\right]$.

This shows that whenever $u(t, x, y)$ is a solution, so is

$\tilde{u}(\tilde{t}, \tilde{x}, \tilde{y})=\frac{1}{2 k_{0}^{2}}\left[2 k_{0}^{2} e^{2 k_{0} \epsilon} u(t, x, y)-k_{0}\left(-1+e^{2 k_{0} \epsilon}\right) g^{\prime}+2\left(e^{k_{0} \epsilon}-1\right)\left(\left(-1+e^{k_{0} \epsilon}\right) h-k_{0} \tilde{y}\right) h^{\prime \prime}\right]$

where

$$
\begin{aligned}
t & =\tilde{t} \\
x & =\frac{1}{2 k_{0}^{2}}\left[e^{-2 k_{0} \epsilon}\left(k_{0}\left(g-e^{2 k_{0} \epsilon}\right) g+2 k_{0} \tilde{x}\right)+2 h^{\prime}\left(-1+e^{k_{0} \epsilon}\right)\left(\left(e^{k_{0} \epsilon}-1\right) h-k_{0} \tilde{y}\right)\right] \\
y & =-\frac{1}{k_{0}}\left[e^{-k_{0} \epsilon}\left(-2 h+2 e^{k_{0} \epsilon} h-k_{0} \tilde{y}\right)\right] .
\end{aligned}
$$

Finally, we are left with the case where $f \neq 0$. This case leads to relatively complicated or too long formulas, and we omit it here.

\section{Classification of low-Dimensional Symmetry algebras}

Group-invariant solutions corresponding to two subgroups which are conjugate under the adjoint action of the symmetry group lie on the same orbit. Therefore, in order to know all the similarity solutions of the ZK equation invariant under $s$-parameter symmetry subgroups, it is sufficient to have a classification of $s$-parameter subalgebras, and an expression for the adjoint representation Ad of $G$. In the case of the ZK equation, as explained in the next section, we shall only need a classification of $s$-parameter subalgebras under the corresponding adjoint action ad of the Lie algebra $L$ of $G$ on itself, where $1 \leq s<3$, and where ad denotes the differential of the $\mathrm{Ad}$. By the connectedness of $G$, in order to find an expression for $\mathrm{Ad}$, we only need to find it for the flow $\operatorname{Ad}\left(\exp \left(\varepsilon V_{i}\right)\right) \cdot \mathbf{v}$ through $\mathbf{v}$ of the oneparameter subgroup $\exp \left(\varepsilon V_{i}\right)$ generated by each basis element $V_{i}$ of $L$. However, if we denote by $V_{\text {ad }}$ the infinitesimal generator of Ad corresponding to each $V \in L$, then $V_{\text {ad }}$ coincides with the Lie bracket on $L: V_{\text {ad }} \cdot \mathbf{v}=[\mathbf{v}, V]$ for all $\mathbf{v} \in L$. We can therefore reconstruct the adjoint representation of the group from that of its Lie algebra, and formulas for $\operatorname{Ad}(\exp (\varepsilon V)) \cdot \mathbf{v}$ based on Lie series or on properties of the flow exist [9, Page 205]. 
For the purpose of finding a representative list of all similarity solutions of the ZK equation, we shall only need as indicated to classify one- and two-dimensional subalgebras. Techniques for performing these classifications can be found in [16, 17. 9. They generally consist in mapping or 'reducing' a system of Lie subalgebra generators to an equivalent system until a canonical representative is achieved. For the ZK symmetry algebra, we shall need the following set of lemmas which are essentially based of the commutation relations (7).

We shall often denote collectively by $\mathbf{w}_{q}$, where $q$ is some function of time, all of the three families of generators $\mathbf{x}_{g}, \mathbf{y}_{h}$ and $\mathbf{z}_{f}$ in (6). It is clear that $\mathbf{w}_{q}$ is a linear function of its argument $q$. When $q$ is a constant function represented by its numeric value $q, \mathbf{w}_{q}$ will be denoted by $\mathbf{w}_{(q)}$. We shall also often represent a general vector of the form (10) simply by its components in terms of $k_{0}, \mathbf{x}_{g}, \mathbf{y}_{h}$ and $\mathbf{z}_{f}$.

$$
\mathbf{v}=k_{0} \mathbf{v}_{0}+\mathbf{x}_{g}+\mathbf{y}_{h}+\mathbf{z}_{f} \equiv\left\{k_{0}, g, h, f\right\}
$$

Lemma 1. Let $f, g$ and $h$ be given functions of $t$, and $k_{0}$ a given constant. Denote by $\alpha$ and $\beta$ some numbers and by $G$ and $H$ some functions of $t$.

(a) For some $\alpha$ and $G, \mathbf{v}_{0}+\mathbf{x}_{g}$ is mapped to $\mathbf{v}_{0}$ under $\operatorname{Ad}\left(\exp \left(\alpha \mathbf{x}_{G}\right)\right)$.

(b) For some $\alpha, \beta, G$ and $H, \mathbf{v}_{0}+\mathbf{x}_{g}+\mathbf{y}_{h}$ is mapped to $\mathbf{v}_{0}$ under $\operatorname{Ad}\left(\exp \left(\alpha \mathbf{x}_{G}\right)\right) \operatorname{Ad}\left(\exp \left(\beta \mathbf{y}_{H}\right)\right)$.

(c) For some $\alpha, \beta, G$ and $H, k_{0} \mathbf{v}_{0}+\mathbf{x}_{g}+\mathbf{y}_{h}+\mathbf{z}_{f}$ is mapped to $k_{0} \mathbf{v}_{0}+\mathbf{z}_{f}$ under $\operatorname{Ad}\left(\exp \left(\alpha \mathbf{x}_{G}\right)\right) \operatorname{Ad}\left(\exp \left(\beta \mathbf{y}_{H}\right)\right)$, provided that $k_{0}^{2}+f^{2} \neq 0$.

(d) For some $\beta$ and $H, \mathbf{x}_{g}+\mathbf{y}_{h}$ is mapped to either $\mathbf{x}_{g}$ or $\mathbf{y}_{h}$ under $\operatorname{Ad}\left(\exp \left(\beta \mathbf{y}_{H}\right)\right)$

(e) For some $\alpha$ and $G, \mathbf{v}_{0}+\mathbf{x}_{g}+\mathbf{y}_{h}$ is mapped to $\mathbf{v}_{0}+\mathbf{y}_{h}$ under $\operatorname{Ad}\left(\exp \left(\alpha \mathbf{x}_{G}\right)\right)$.

(f) For some $\alpha$ and $G, k_{0} \mathbf{v}_{0}+\mathbf{x}_{g}+\mathbf{y}_{h}+\mathbf{z}_{f}$ is mapped to $k_{0} \mathbf{v}_{0}+\mathbf{y}_{h}+\mathbf{z}_{f}$ under $\operatorname{Ad}\left(\exp \left(\alpha \mathbf{x}_{G}\right)\right)$, provided that $k_{0}^{2}+f^{2} \neq 0$.

Proof. We have $\operatorname{Ad}\left(\exp \left(\alpha \mathbf{x}_{G}\right)\right)\left(\mathbf{v}_{0}\right)=\mathbf{v}_{0}-2 \alpha \mathbf{x}_{G}$, and $\operatorname{Ad}\left(\exp \left(\alpha \mathbf{x}_{G}\right)\right)\left(\mathbf{v}_{0}+\mathbf{x}_{g}\right)=$ $\mathbf{v}_{0}-2 \alpha \mathbf{x}_{G}+\mathbf{x}_{g}$, which has component $\{1, g-2 \alpha G, 0,0\}$. It suffices then to choose $G=g /(2 \alpha)$ with $\alpha \neq 0$, which proves part (a). For part (b), we noticed that when $h$ is zero, the statement reduces to that of part (a). Otherwise, $V=\mathbf{v}_{0}+\mathbf{x}_{g}+\mathbf{y}_{h}$ is transformed under $\operatorname{Ad}\left(\exp \left(\beta \mathbf{y}_{H}\right)\right)$ to a vector with component $\left\{1, g+(\beta / 2)\left(-H h^{\prime}+h H^{\prime}\right), h-\beta H, 0\right\}$. If we therefore choose $H=h / \beta$ to cancel the term $\mathbf{y}_{h}$ in $V$, the result will follow from that of part (a). For (c), we note that if $f=0$, the problem reduces to that of part $(b)$. Thus we first assume that $f \neq 0$, and $k_{0}=0$. Then $\operatorname{Ad}\left(\exp \left(\beta \mathbf{y}_{H}\right)\right)$ will transform $V=\mathbf{x}_{g}+\mathbf{y}_{h}+\mathbf{z}_{f}$ into a vector $V^{(1)}$ of the form $\left\{0, g_{1}, h+\beta\left(-\frac{2}{3} H f^{\prime}+f H^{\prime}\right), f\right\}$, where $g_{1}$ is a certain function of $t$. Thus to cancel the term $\mathbf{y}_{h}$ in $V$, it suffices to choose $H$ as a solution to the equation $h+\beta\left(-\frac{2}{3} H f^{\prime}+f H^{\prime}\right)=0$. Notice that this is a linear first order differential equation of the general form

$$
K^{\prime}(t)=Q(t, K(t))
$$

where $Q$ is a linear function of the unknown function $K$, and such linear equations always have a solution. For instance, the latter equation for $H$ has solution

$$
H(t)=C f(t)^{2 / 3}+f(t)^{2 / 3} \int_{1}^{t}-\frac{h(s)}{\beta f(s)} d s, \quad(\beta \neq 0),
$$

where $C$ is an arbitrary constant. Now suppose that $V^{(1)}$ reduces to a vector of the form $\left\{0, g_{1}^{0}, 0, f\right\}$ when $H$ is so chosen that the third component of $V^{1}$ vanishes. 
Then $\operatorname{Ad}\left(\exp \left(\alpha \mathbf{x}_{G}\right)\right)$ will map the resulting vector $V^{(1)}$ to

$$
V^{(2)}=\left\{0, g-\alpha\left(-(1 / 3) G f^{\prime}+f G^{\prime}\right), 0, f\right\}
$$

The condition that the second component of $V^{(2)}$ vanishes is a linear equation in $G$ of the form (12), showing that we can reduce $V$ to $\mathbf{x}_{f}=k_{0} \mathbf{v}_{0}+\mathbf{x}_{f}$. If on the other hand both $k_{0}$ and $f$ are nonzero, then $\operatorname{Ad}\left(\exp \left(\beta \mathbf{y}_{H}\right)\right)$ will map $V=k_{0} \mathbf{v}_{0}+\mathbf{x}_{g}+$ $\mathbf{y}_{h}+\mathbf{z}_{f}$ to a vector $V^{(1)}$ with component $\left\{k_{0}, g_{1}, h+\beta\left(-\frac{H}{3}\left(3+2 f^{\prime}\right)+f H^{\prime}\right), f\right\}$, for some function $g_{1}$ depending on $H$. Let $H_{0}$ be a function for which the third component of $V^{(1)}$ vanishes, and denote again by $V^{(1)}=\left\{k_{0}, g_{1}^{0}, 0, f\right\}$ the corresponding image of $V$ under $\operatorname{Ad}\left(\exp \left(\beta \mathbf{y}_{H_{0}}\right)\right)$. Then,

$$
\operatorname{Ad}\left(\exp \left(\alpha \mathbf{x}_{G}\right)\right)\left(V^{(1)}\right)=\left\{k_{0}, g-(1 / 3) \alpha\left(G\left(6+f^{\prime}\right)-3 f G^{\prime}\right), f\right\}
$$

showing that we can map $V$ as indicated to $k_{0} \mathbf{v}_{0}+\mathbf{z}_{f}$, and this proves (c).

For (d), if $h=0$, then $V=\mathbf{x}_{g}+\mathbf{y}_{h}$ reduces to $\mathbf{x}_{g}$ and we choose $\beta=0$, otherwise it suffices to choose $H$ as a solution to the linear equation

$$
g+\frac{1}{2} \beta\left(-H h^{\prime}+h H^{\prime}\right)=0
$$

in order to map $V$ to $\mathbf{y}_{h}$. For (e), it suffices to choose $G=g /(2 \alpha)$, with $\alpha \neq 0$. To prove (f), we simply note that if $k_{0}=0$, we let $G$ be the any solution to the linear ODE

$$
g-\alpha\left(\frac{1}{3} G f^{\prime}+f G^{\prime}\right)=0, \quad(\alpha \neq 0) .
$$

If $f=0$, we let $G$ be a solution to $g-2 \alpha G=0$, and if both $k_{0}$ and $f$ are nonzero, we let $G$ be the solution to the linear oDE

$$
g-\alpha\left(\frac{1}{3} G\left(6+f^{\prime}\right)-3 f G^{\prime}\right)=0, \quad(\alpha \neq 0) .
$$

This completes the proof of Lemma 1

Lemma 2. Let $g, h$ and $f$ be given functions of time. Then for any $\varepsilon \in \mathbb{R}$, and for every nonzero function $K=K(t)$, we have

(a)

$$
\begin{aligned}
& \operatorname{Ad}\left(\exp \left(\varepsilon \mathbf{z}_{K}\right)\right)\left(\mathbf{x}_{g}\right)=\mathbf{x}_{G(t, \varepsilon)}, \quad \operatorname{Ad}\left(\exp \left(\varepsilon \mathbf{z}_{K}\right)\right)\left(\mathbf{y}_{h}\right)=\mathbf{y}_{H(t, \varepsilon)}, \\
& \operatorname{Ad}\left(\exp \left(\varepsilon \mathbf{z}_{K}\right)\right)\left(\mathbf{z}_{f}\right)=\mathbf{z}_{F(t, \varepsilon)},
\end{aligned}
$$


where the functions $G(t, \varepsilon), H(t, \varepsilon)$ and $F(t, \varepsilon)$ are given by

$$
\begin{aligned}
G(t, \varepsilon) & =K(t)^{1 / 3} Q_{1}\left(\varepsilon-\int_{1}^{t} \frac{d s}{K(s)}\right), \text { and } Q_{1} \text { satisfies } \\
g(t) & =K(t)^{1 / 3} Q_{1}\left(-\int_{1}^{t} \frac{d s}{K(s)}\right) . \\
H(t, \varepsilon) & =K(t)^{2 / 3} Q_{2}\left(\varepsilon-\int_{1}^{t} \frac{d s}{K(s)}\right), \text { and } Q_{2} \text { satisfies } \\
h(t) & =K(t)^{2 / 3} Q_{2}\left(-\int_{1}^{t} \frac{d s}{K(s)}\right) . \\
F(t, \varepsilon) & =K(t) Q_{3}\left(\varepsilon-\int_{1}^{t} \frac{d s}{K(s)}\right), \text { and } Q_{3} \text { satisfies } \\
f(t) & =K(t) Q_{3}\left(-\int_{1}^{t} \frac{d s}{K(s)}\right) .
\end{aligned}
$$

(b) Let $\mathbf{w}_{q}$, where $q=q(t)$, denote collectively all generators of the form $\mathbf{x}_{g}, \mathbf{y}_{h}$ and $\mathbf{z}_{f}$. Then, whenever $q \neq 0$, there exists a function $K$ such that $\operatorname{Ad}\left(\exp \left(\varepsilon \mathbf{z}_{K}\right)\right) \mathbf{w}_{q}=\mathbf{w}_{(1)}$.

Proof. From Equation (77) we have $\left[\mathbf{z}_{K}, \mathbf{x}_{g}\right]=\mathbf{x}_{\left(K g^{\prime}-K^{\prime} g / 3\right)}$, and this shows that if we express $\operatorname{Ad}\left(\exp \left(\varepsilon \mathbf{z}_{K}\right)\right)\left(\mathbf{x}_{g}\right)$ in terms of the Lie series and use the linearity of $\mathbf{x}_{g}$ as a function of its argument $g$, then $\operatorname{Ad}\left(\exp \left(\varepsilon \mathbf{z}_{K}\right)\right)\left(\mathbf{x}_{g}\right)$ must be of the form $\mathbf{x}_{G(t, \varepsilon)}$ for a certain function $G=G(t, \varepsilon)$. Now, the expression for $G(t, \varepsilon)$ follows from the properties of the flow $\operatorname{Ad}\left(\exp \left(\varepsilon \mathbf{z}_{K}\right)\right) \mathbf{x}_{g}$ of Ad through $\mathbf{x}_{g}$ under the one-parameter subgroup $\exp \left(\varepsilon \mathbf{z}_{K}\right)$ generated by $K$. The expressions for $\operatorname{Ad}\left(\exp \left(\varepsilon \mathbf{z}_{K}\right)\right)\left(\mathbf{y}_{h}\right)$ and $\operatorname{Ad}\left(\exp \left(\varepsilon \mathbf{z}_{K}\right)\right)\left(\mathbf{z}_{f}\right)$ and for the corresponding functions $H(t, \varepsilon)$ and $F(t, \varepsilon)$ are derived in a similar way, and this proves (a).

Part (b) simply says that if for instance we have $g \neq 0$, then we can choose the function $K(t)$ in such a way that the resulting function $G(t, \varepsilon)$ in (13) is 1 , and that the same holds for both $h$ and $f$ and for the corresponding functions $H(t, \varepsilon)$ and $F(t, \varepsilon)$ in (13). The existence of such functions $K$ is just a consequence of a result of Neuman [19]. A proof of the existence of $K$ is also given in 11. This completes the proof of the lemma.

3.1. Classification of one-dimensional subalgebras. To implement this classification, we denote as usual by $\mathbf{v}=k_{0} \mathbf{v}_{0}+\mathbf{x}_{g}+\mathbf{y}_{h}+\mathbf{z}_{f}$ a general nonzero vector in $L$, and for $\mathbf{v}_{1}, \mathbf{v}_{2} \in L$ we write $\mathbf{v}_{1} \sim \mathbf{v}_{2}$ if $\mathbf{v}_{2}=\operatorname{Ad}_{B}\left(\mathbf{v}_{1}\right)$, for some group element $B$ in $G$. When both $k_{0}$ and $f$ equal zero, we have $\mathbf{v} \sim \mathbf{x}_{g}$ or $\mathbf{v} \sim \mathbf{y}_{h}$ by part (e) of Lemma 1. Otherwise, we have $k_{0}^{2}+f^{2} \neq 0$, and hence $\mathbf{v} \sim k_{0} \mathbf{v}_{0}+\mathbf{z}_{f}$ by part (c) of Lemma 1. Thus every one-dimensional subalgebra of $L$ is equivalent under the adjoint representation to either $\mathbf{x}_{g}, \mathbf{y}_{h}$ or $k_{0} \mathbf{v}_{0}+\mathbf{z}_{f}$. Since we have $\operatorname{Ad}_{B}\left(k_{0} \mathbf{v}_{0}+\mathbf{z}_{f}\right)=k_{0} \mathbf{v}_{0}+A d_{B}\left(\mathbf{z}_{f}\right)$, for every group element $B$, it follows from Lemma 2 that if $f=0$, then $\left(k_{0} \mathbf{v}_{0}+\mathbf{z}_{f}\right) \sim \mathbf{v}_{0}$, other wise $\left(k_{0} \mathbf{v}_{0}+\mathbf{z}_{f}\right) \sim k_{0} \mathbf{v}_{0}+\mathbf{z}_{(1)}$. The same lemma thus implies that every one dimensional subalgebra of the ZK symmetry algebra is equivalent under the adjoint representation to one of the following Lie algebras 


$$
\mathcal{L}_{1,1}=\left\{\mathbf{x}_{(1)}\right\}, \quad \mathcal{L}_{1,2}=\left\{\mathbf{y}_{(1)}\right\}, \quad \mathcal{L}_{1,3}=\left\{\mathbf{v}_{0}\right\} \quad \text { or } \quad \mathcal{L}_{1,4}=\left\{k_{0} \mathbf{v}_{0}+\mathbf{z}_{(1)}\right\} .
$$

Note that according to (6), we have

$$
\mathbf{x}_{(1)}=\partial_{x}, \quad \mathbf{y}_{(1)}=\partial_{y}, \quad \text { and } \quad \mathbf{z}_{(1)}=\partial_{t},
$$

which shows that the canonical forms thus obtained are very simplified. To see why these four Lie algebras are non equivalent, we first note that $\operatorname{Ad}\left(\exp \left(\varepsilon \mathbf{v}_{0}\right)\right)$ acts only diagonally, by scalling its argument, while for every function $F=F(t)$, $\operatorname{Ad}\left(\exp \left(\varepsilon \mathbf{z}_{F}\right)\right)$ leaves $\mathbf{v}_{0}$ invariant and maps, by Lemma $2, \mathbf{x}_{g}, \mathbf{y}_{h}$ and $\mathbf{z}_{f}$ to $\mathbf{x}_{\mathfrak{g}}, \mathbf{y}_{\mathfrak{h}}, \mathbf{z}_{\mathfrak{f}}$, respectively, for some functions $\mathfrak{g}=\mathfrak{g}(t, \varepsilon), \mathfrak{h}=\mathfrak{h}(t, \varepsilon)$ and $\mathfrak{f}=\mathfrak{f}(t, \varepsilon)$. On the other hand, if we set

$$
\mathcal{F}=\operatorname{Ad}\left(\exp \left(\alpha \mathbf{x}_{G}\right)\right) \operatorname{Ad}\left(\exp \left(\beta \mathbf{y}_{H}\right)\right)
$$

then we have,

$$
\mathcal{F}\left(\mathbf{v}_{0}\right)=\{1,-2 \alpha G,-\beta H, 0\} \sim \mathbf{v}_{0}
$$

and

$$
\begin{aligned}
\mathcal{F}\left(k_{0} \mathbf{v}_{0}+\mathbf{z}_{(1)}\right) & =\left\{k_{0} \mathbf{v}_{0}, \alpha\left(-2 G+G^{\prime}\right)+(1 / 4) \beta^{2}\left(H^{\prime 2}-H H^{\prime \prime}\right), \beta\left(-H+H^{\prime}\right), 1\right\} \\
& \sim k_{0} \mathbf{v}_{0}+\mathbf{z}_{(1)} .
\end{aligned}
$$

We also have $\mathcal{F}\left(\mathbf{x}_{(1)}\right)=\mathbf{x}_{(1)}$, and $\mathcal{F}\left(\mathbf{y}_{(1)}\right)=\mathbf{y}_{(1)}$. This shows that the four Lie algebras thus obtained are non-equivalent under the adjoint representation of $G$, and thus completes the classification of one-dimensional subalgebras of $L$.

3.2. Classification of two-dimensional subalgebras. Every two dimensional subalgebra $L_{2}$ of $L$ is solvable and if we denote by $\mathcal{B}=\left\{V_{1}, V_{2}\right\}$ a basis of $L_{2}$, then either $L_{2}$ is abelian and thus $\left[V_{1}, V_{2}\right]=0$, or $L_{2}$ has a nonzero nilradical, in which case its commutation relations can be put in the form $\left[V_{1}, V_{2}\right]=V_{1}$. To classify two dimensional subalgebras of $L$, we let $V_{1}$ be in one of the canonical forms (15), while $V_{2}=\mathbf{v}$ is a general vector of the form (11). By the possible forms for $V_{1}$ given in (15), we shall therefore have to consider four basic cases for $\mathcal{B}$. To reduce $L_{2}$ to a canonical form, we transform it under maps of the form $\operatorname{Ad}_{B}$, for appropriately chosen group elements $B$ in the symmetry group $G$, and also make use of the commutation relations in $L_{2}$ to obtain some restrictions on the functions $g, h, f$ and the free parameter $k_{0}$ defining $V_{2}=\mathbf{v}$. If $\mathcal{B}_{1}$ is a basis of a subalgebra $S_{1}$ and $\mathcal{B}_{2}$ a basis of a subalgebra $S_{2}$, we write $\mathcal{B}_{1} \sim \mathcal{B}_{2}$ if $S_{2}=\operatorname{Ad}_{B}\left(S_{1}\right)$ for some $B \in G$. In this subsection, we shall let $c_{1}, c_{2}$ and $c_{3}$ denote three arbitrary constants.

\subsubsection{Abelian subalgebras.}

Case (a): $\mathcal{B}=\left\{\mathbf{v}_{0}, \mathbf{v}\right\}$.

The condition $\left[\mathbf{v}_{0}, \mathbf{v}\right]=0$, implies that $g=h=0$. Thus $\mathbf{v}=k_{0} \mathbf{v}_{0}+\mathbf{z}_{f}$. Since for every $\varepsilon \in \mathbb{R}$ and every function $K$ we have

$$
\operatorname{Ad}\left(\exp \left(\varepsilon \mathbf{z}_{K}\right)\right)\left(k_{0} \mathbf{v}_{0}+\mathbf{z}_{f}\right)=k_{0} \mathbf{v}_{0}+\operatorname{Ad}\left(\exp \left(\varepsilon \mathbf{z}_{K}\right)\right)\left(\mathbf{z}_{f}\right)
$$

and since $\operatorname{Ad}\left(\exp \left(\varepsilon \mathbf{z}_{K}\right)\right) \mathbf{v}_{0}=\mathbf{v}_{0}$, it follows that if $f=0$, then $\mathbf{v} \sim \mathbf{v}_{0}$. Otherwise, $\mathbf{v} \sim k_{0} \mathbf{v}_{0}+\mathbf{z}_{(1)}$. Since $L_{2}$ is two-dimensional, we must have $\mathcal{B} \sim\left\{\mathbf{v}_{0}, k_{0} \mathbf{v}_{0}+\mathbf{z}_{(1)}\right\}$.

Case (b): $\quad \mathcal{B}=\left\{k_{1} \mathbf{v}_{0}+\mathbf{z}_{(1)}, \mathbf{v}\right\}, k_{1} \in \mathbb{R}$. 
We must have in this case

$$
g=c_{1} e^{2 k_{1} t}, \quad h=c_{2} e^{k_{1} t}, \quad \text { and } f=c_{3} .
$$

Thus $\mathcal{B} \sim\left\{k_{1} \mathbf{v}_{0}+\mathbf{z}_{(1)}, \mathbf{v}\right\}$, where the components $g, h, f$ of $\mathbf{v}$ are given by (17).

Case (c): $\mathcal{B}=\left\{\mathbf{x}_{(1)}, \mathbf{v}\right\}$.

We must have $f=6 k_{0} t+c_{3}$. If $k_{0}=0$ and $c_{3}=0$, then by part (d) of Lemma 1, under $\operatorname{Ad}\left(\exp \left(\beta \mathbf{y}_{H}\right)\right)$, we have $\mathbf{v} \sim \mathbf{x}_{g}$ or $\mathbf{v} \sim \mathbf{y}_{h}$, and thus, since $\operatorname{Ad}\left(\exp \left(\beta \mathbf{y}_{H}\right)\right) \mathbf{x}_{g}=\mathbf{x}_{g}$ for all $g$, we have $\mathcal{B} \sim\left\{\mathbf{x}_{(1)}, \mathbf{x}_{g}\right\}$ or $\mathcal{B} \sim\left\{\mathbf{x}_{(1)}, \mathbf{y}_{h}\right\}$. If $k_{0} \neq 0$ or $c_{3} \neq 0$, then by part (c) of Lemma 1 we have $\mathbf{v} \sim k_{0} \mathbf{v}_{0}+\mathbf{z}_{\left(6 k_{0} t+c_{3}\right)}$, and hence

$$
\mathcal{B} \sim\left\{\mathbf{x}_{(1)}, k_{0} \mathbf{v}_{0}+\mathbf{z}_{\left(6 k_{0} t+c_{3}\right)}\right\} .
$$

Case (d): $\mathcal{B}=\left\{\mathbf{y}_{(1)}, \mathbf{v}\right\}$.

We must have $f=-(3 / 2) k_{0} t+c_{3}$, and $h=c_{2}$. Thus if $k_{0}=0$ and $c_{3}=0$, then $\mathbf{v} \sim \mathbf{x}_{g}+\mathbf{y}_{\left(c_{2}\right)}$, and no further reduction of the basis $\mathcal{B}=\left\{\mathbf{y}_{(1)}, \mathbf{x}_{g}+\mathbf{y}_{\left(c_{2}\right)}\right\}$ is possible. If $k_{0} \neq 0$ or $c_{3} \neq 0$, then

$$
\mathcal{B} \sim\left\{\mathbf{y}_{(1)}, k_{0} \mathbf{v}_{0}+\mathbf{y}_{\left(c_{2}\right)}+\mathbf{z}_{\left(-(3 / 2) k_{0} t+c_{3}\right)}\right\} .
$$

This is because by part (f) of Lemma 1, we have $\mathbf{v} \sim k_{0} \mathbf{v}_{0}+\mathbf{y}_{c_{2}}+\mathbf{z}_{f}$, under $\operatorname{Ad}\left(\exp \left(\alpha \mathbf{x}_{G}\right)\right)$, and the operator $\operatorname{Ad}\left(\exp \left(\alpha \mathbf{x}_{G}\right)\right)$ leaves $\mathbf{y}_{(1)}$ invariant for every $\alpha$ and $G$. Again, all the canonical representatives of two-dimensional subalgebras thus obtained are clearly pairwise nonequivalent, and this completes the classification problem in the abelian case.

\subsubsection{Non-abelian subalgebras.}

Case (a): $\mathcal{B}=\left\{\mathbf{v}_{0}, \mathbf{v}\right\}$ or $\mathcal{B}=\left\{k_{1} \mathbf{v}_{0}+\mathbf{z}_{(1)}, \mathbf{v}\right\}, \quad k_{1} \in \mathbb{R}$.

Since $\mathbf{v}_{0}$ is not in the derived subalgebra of $L$, the case $\mathcal{B}=\left\{\mathbf{v}_{0}, \mathbf{v}\right\}$ cannot occur, while the case $\mathcal{B}=\left\{k_{1} \mathbf{v}_{0}+\mathbf{z}_{(1)}, \mathbf{v}\right\}$ occurs only if $k_{1}=0$, in which case we must have

$$
g=c_{1}, \quad h=c_{2}, \quad \text { and } \quad f=t+c_{3}
$$

Consequently, in this case

$$
\mathcal{B} \sim\left\{\mathbf{z}_{(1)}, k_{0} \mathbf{v}_{0}+\mathbf{x}_{\left(c_{1}\right)}+\mathbf{y}_{\left(c_{2}\right)}+\mathbf{z}_{\left(t+c_{3}\right)}\right\} .
$$

Case (b): $\mathcal{B}=\left\{\mathbf{x}_{(1)}, \mathbf{v}\right\}$.

We must have in this case $f=\left(3-6 k_{0}\right) t+c_{3}$. Thus if $k_{0}=1 / 2$ and $c_{3}=0$, then by Lemma 1 part (b), $\operatorname{Ad}\left(\exp \left(\alpha \mathbf{x}_{G}\right)\right) \operatorname{Ad}\left(\exp \left(\beta \mathbf{y}_{H}\right)\right)$ will map $\mathbf{v}$ to $\mathbf{v}_{0}$ and leave $\mathbf{x}_{(1)}$ unchanged. Hence, $\mathcal{B} \sim\left\{\mathbf{x}_{(1)}, \mathbf{v}_{0}\right\}$ in this case. If either $k_{0} \neq 1 / 2$ or $c_{3} \neq 0$, the same lemma implies that $\mathbf{v} \sim k_{0} \mathbf{v}_{0}+\mathbf{z}_{f}$, under a similar transformation that leaves $\mathbf{x}_{(1)}$ unchanged. Consequently, in this second case we have $\mathcal{B} \sim\left\{\mathbf{x}_{(1)}, k_{0} \mathbf{v}_{0}+\mathbf{z}_{\left(\left(3-6 k_{0}\right) t+c_{3}\right)}\right\}$.

Case (c): $\mathcal{B}=\left\{\mathbf{y}_{(1)}, \mathbf{v}\right\}$.

The commutation relations imply that $f=(3 / 2)\left(1-k_{0}\right) t+c_{3}$, and $h=c_{2}$. Thus if $k_{0}=1$ and $c_{3}=0$, by part (e) of Lemma 1. $\operatorname{Ad}\left(\exp \left(\alpha \mathbf{x}_{G}\right)\right)$ maps $\mathbf{v}$ to $\mathbf{v}_{0}+\mathbf{y}_{\left(c_{2}\right)}$. Since this operator leaves $\mathbf{y}_{(1)}$ invariant, we must have $\mathcal{B} \sim\left\{\mathbf{y}_{(1)}, \mathbf{v}_{0}+\mathbf{y}_{\left(c_{2}\right)}\right\}$ in 
this case. If $k_{0} \neq 1$ or $c_{3} \neq 0$, part (f) of the same lemma shows that $\operatorname{Ad}\left(\exp \left(\alpha \mathbf{x}_{G}\right)\right)$ maps $\mathbf{v}$ to $k_{0} \mathbf{v}_{0}+\mathbf{y}_{\left(c_{2}\right)}+\mathbf{z}_{f}$. Consequently,

$$
\mathcal{B} \sim\left\{\mathbf{y}_{(1)}, k_{0} \mathbf{v}_{0}+\mathbf{y}_{\left(c_{2}\right)}+\mathbf{z}_{\left((3 / 2)\left(1-k_{0}\right) t+c_{3}\right)}\right\} .
$$

Since the canonical forms thus obtained are non-equivalent by construction, this completes the classification of two-dimensional subalgebras of $L$ in the non-abelian case.

We have thus obtained the following list of canonical forms of non-equivalent two-dimensional subalgebras of $L$, in which $k_{0}, k_{1}, c_{1}, c_{2}$, and $c_{3}$ are free parameters, unless otherwise specified.

(1) Abelian subalgebras

$$
\begin{aligned}
\mathcal{L}_{2,1} & =\left\{\mathbf{v}_{0}, k_{0} \mathbf{v}_{0}+\mathbf{z}_{(1)}\right\} \\
\mathcal{L}_{2,2} & =\left\{k_{1} \mathbf{v}_{0}+\mathbf{z}_{(1)}, k_{0} \mathbf{v}_{0}+\mathbf{x}_{\left(c_{1} e^{2 k_{1} t}\right)}+\mathbf{y}_{\left(c_{2} e^{k_{1} t}\right)}+\mathbf{z}_{\left(c_{3}\right)}\right\} \\
\mathcal{L}_{2,3} & =\left\{\mathbf{x}_{(1)}, \mathbf{x}_{g}\right\}, \quad\left(g^{\prime} \neq 0\right) \\
\mathcal{L}_{2,4} & =\left\{\mathbf{x}_{(1)}, \mathbf{y}_{h}\right\} \\
\mathcal{L}_{2,5} & =\left\{\mathbf{x}_{(1)}, k_{0} \mathbf{v}_{0}+\mathbf{z}_{\left(6 k_{0} t+c_{3}\right)}\right\}, \quad k_{0}^{2}+c_{3}^{2} \neq 0 \\
\mathcal{L}_{2,6} & =\left\{\mathbf{y}_{(1)}, \mathbf{x}_{g}+\mathbf{y}_{\left(c_{2}\right)}\right\} \\
\mathcal{L}_{2,7} & =\left\{\mathbf{y}_{(1)}, k_{0} \mathbf{v}_{0}+\mathbf{y}_{\left(c_{2}\right)}+\mathbf{z}_{\left((-3 / 2)\left(k_{0} t+c_{3}\right)\right)}\right\}, \quad k_{0}^{2}+c_{3}^{2} \neq 0
\end{aligned}
$$

(2) Non-abelian subalgebras

$$
\begin{aligned}
\mathcal{L}_{2,8} & =\left\{\mathbf{z}_{(1)}, k_{0} \mathbf{v}_{0}+\mathbf{x}_{\left(c_{1}\right)}+\mathbf{y}_{\left(c_{2}\right)}+\mathbf{z}_{\left(t+c_{3}\right)}\right\} \\
\mathcal{L}_{2,9} & =\left\{\mathbf{x}_{(1)}, \mathbf{v}_{0}\right\} \\
\mathcal{L}_{2,10} & =\left\{\mathbf{x}_{(1)}, k_{0} \mathbf{v}_{0}+\mathbf{z}_{\left(\left(3-6 k_{0}\right) t+c_{3}\right)}\right\}, \quad\left(k_{0}-\frac{1}{2}\right)^{2}+c_{3}^{2} \neq 0 \\
\mathcal{L}_{2,11} & =\left\{\mathbf{y}_{(1)}, \mathbf{v}_{0}+\mathbf{y}_{\left(c_{2}\right)}\right\} \\
\mathcal{L}_{2,12} & =\left\{\mathbf{y}_{(1)}, k_{0} \mathbf{v}_{0}+\mathbf{y}_{\left(c_{2}\right)}+\mathbf{z}_{\left((3 / 2)\left(1-k_{0}\right) t+c_{3}\right)}\right\}, \quad\left(k_{0}-1\right)^{2}+c_{3}^{2} \neq 0
\end{aligned}
$$

\section{Similarity Reductions of the ZK equation}

We turn our attention in this section to the problem of finding the group-invariant solutions of the ZK equation. By group invariant solutions we mean solutions which are invariant by the group transformations in the sense that, roughly speaking, each of them is transformed into itself by every group transformation. In other words, their graph is a locally $G$-invariant subset. In the case of a PDE such solutions can be found by solving a differential equation in fewer independent variables. We shall therefore seek a reduction of the ZK equation to $(1+1)$-dimensional equations or to ordinary differential equations. To reduce the equation to one with $s$ fewer independent variables, the general procedure [9] is to look for subgroups of the full symmetry group whose orbits have dimension $s$. Each such subgroup yields a set of $3-s$ functionally independent invariants which can be written in the form $\xi=\eta(\mathbf{w})$, where $\mathbf{w}=(t, x, y)$, in addition to another functionally independent invariant of the form $w=\zeta(\mathbf{w}, u)$. We must clearly have $1 \leq s<3$, since the ZK equation has exactly three independent variables. Solving this last equality for the solution $u$ of the original equation shows that $u$ will always be determined in the case of the ZK 
equation by some equations of the form

$$
u(t, x, y)=\alpha+\beta w(\xi), \quad \xi=\eta(\mathbf{w})
$$

where $\alpha$ and $\beta$ are functions of $\mathbf{w}$. Equation (18) can be used to rewrite the original equation in terms of the invariant functions $\xi=\eta(\mathbf{w})$, and $w$ considered as new variables, and this yields the reduced system in only $3-s$ independent variables 9. Expressing the solution of the reduced equation in the form $w=F(\xi)$, and substituting in this last equality the expressions for $w$ and $\xi$ from equation (18), the solution $u$ of the original equation can be found. We treat separately reductions by one dimensional subgroups and reductions by two dimensional subgroups.

4.1. Reduction by one-dimensional subgroups. As already indicated, a reduction by one dimensional subgroups will yield a partial differential equation in only $2=3-1$ independent variables. The generic form of the infinitesimal generator of such a group is given by (10). In the case of a reduction by one-dimensional subgroups, the function the $\xi=\eta(\mathbf{w})$ in (18) will have the form $\xi=\left(\eta_{1}, \eta_{2}\right)$, and for simplicity of notation we shall set $r=\eta_{1}$ and $z=\eta_{2}$. In order to completely define a reduced equation, we will only need to give the explicit formulas for $u, z$, and $r$ corresponding to Equation (18) and the reduced equation itself. We first give reductions based on the classification of one-dimensional subalgebras given in (15), and then reductions based of a direct case analysis afterward.

4.1.1. Reductions based on one-dimensional subalgebras classification. This procedure consists in finding the reduced equation corresponding to each of the four non-equivalent canonical forms of one-dimensional subalgebras of $L$ given in (15). The list of all solutions to the four resulting reduced equations should represent an optimal list of 1-parameter group invariant solutions, with the property that every other 1-parameter group invariant solution of the ZK equation can be mapped to precisely one solution in the list via the adjoint representation of $G$.

(1) Reduction by $\mathcal{L}_{1,1}=\left\{\mathbf{x}_{(1)}\right\}$.

For $\mathbf{x}_{(1)}=\partial_{x}$, the reduction formula is simply $u=u(t, y), r=t$, and $z=y$. The reduced equation is the linear equation

$$
u_{y, y}=0
$$

with solution $u=q_{1} y+q_{2}$, where $q_{1}$ and $q_{2}$ are arbitrary functions of $t$.

(2) Reduction by $\mathcal{L}_{1,2}=\left\{\mathbf{y}_{(1)}\right\}$.

For $\mathbf{y}_{(1)}=\partial_{y}$, the reduction formula is $u=u(t, x), r=t, z=x$. The reduced equation is

$$
-u_{x}^{2}+u_{t, x}-u u_{x, x}=0 .
$$

(3) Reduction by $\mathcal{L}_{1,3}=\left\{\mathbf{v}_{0}\right\}$.

The reduction formula is

$$
u=x w(t, z), \quad r=t, \quad z=y^{2} / 2,
$$

and the corresponding reduced equation is

$$
w_{r}-2 w_{z}-\left(w-z w_{z}\right)^{2}-z w_{r, z}-4 z w_{z, z}-z^{2} w w_{z, z}=0 .
$$


(4) Reduction by $\mathcal{L}_{1,4}=\left\{k_{0} \mathbf{v}_{0}+\mathbf{z}_{(1)}\right\}$.

The reduction formula is

$$
u=x w(r, z), \quad r=2 k_{0} t-\ln (x), \quad z=y^{2} / x,
$$

which gives rise to the reduced equation

$$
\begin{aligned}
2 w_{z} & +\left(-w+w_{r}+z w_{z}\right)^{2}+2 k_{0}\left(w_{r, r}+z w_{r, z}\right)+4 z w_{z, z} \\
& +w\left(-w_{r}+w_{r, r}+z\left(2 w_{r, z}+z w_{z, z}\right)\right)-2 k_{0} w_{r}=0 .
\end{aligned}
$$

For $k_{0}=0$, this last equation corresponds to the much simpler reduction by $\left\{\mathbf{z}_{(1)}\right\}$ given by

$$
u_{x}^{2}+u u_{x, x}+u_{y, y}=0 .
$$

4.1.2. Reduction by direct case analysis. This procedure consists in obtaining all possible reductions of the ZK equation by one-parameter subgroups, by a suitable consideration of each relevant case separately. Although it gives directly all possible cases of reduced equations, it might involve tedious or too long calculations in certain cases.

Case 1: $g=h=f=0$, and $k_{0} \neq 0$

This is just a reduction by the subgroup generated by $\mathbf{v}_{0}$. The reduction formula and corresponding reduced equations are already given in (19) and (20).

Case 2 : $h=f=0$, and $g \neq 0$, and $k_{0}$ arbitrary

Case 2a : $k_{0}=0$

In this case we have

$$
u=\frac{g^{\prime}}{g}(w-x), \quad r=t, \quad z=y .
$$

The reduced equation is

$$
g^{\prime \prime}+g^{\prime} w_{z, z}=0
$$

This gives rise to the invariant solution

$$
u=\frac{g^{\prime}}{g}(w-x), \quad w=a(t) y+b(t), \quad g(t)=a_{1} t+a_{2}
$$

where $a(t), b(t)$ are arbitrary functions, while $a_{1}$ and $a_{2}$ are arbitrary constants.

Case $2 \mathrm{~b}: k_{0} \neq 0$

The reduction formula is

$$
u=y^{2} w(t, z)+\frac{g^{\prime}}{2 a}, \quad r=t, \quad z=\frac{1}{y^{2}}\left(x+g /\left(2 k_{0}\right)\right),
$$

and the reduced equation is

$$
-2 w+2 z w_{z}-w_{z}^{2}+w_{z, t}-\left(w+4 z^{2}\right) w_{z, z}=0 .
$$

Case 3 : $f=0$, and $h \neq 0$

Case 3a : $k_{0}=0$

The reduction formula is

$$
u=\frac{1}{4 h}\left(w(t, z)-\left(2 y g^{\prime}+y^{2} h^{\prime \prime}\right)\right), \quad r=t, \quad z=x-\frac{y}{2 h}\left(g+y h^{\prime} / 2\right) .
$$


The reduced equation is the system

$$
\begin{aligned}
h^{\prime} w_{z}+h^{\prime \prime} & =0 \\
w_{z, z} & =0 \\
-w_{z}^{2}+4 w_{z, t}-w w_{z, z} & =0 .
\end{aligned}
$$

This system implies that

$$
w=\frac{z}{-t+a_{1}}+a(t), \quad h=\frac{a_{2}}{2} t^{2}+a_{1} a_{2} t+a_{3},
$$

and the corresponding invariant solution is

$u=\frac{-t\left(2 a_{1}+t\right) x a_{2}-2 x a_{3}+a(t)\left(a_{1}+t\right)\left(t\left(2 a_{1}+t\right) a_{2}+2 a_{3}\right)+y\left(g+\left(-a_{1}-t\right) g^{\prime}\right)}{\left(a_{1}+t\right)\left(t\left(2 a_{1}+t\right) a_{2}+2 a_{3}\right)}$,

where $a(t)$ is an arbitrary function, while $a_{1}, a_{2}$ and $a_{3}$ are arbitrary constants.

Case $3 \mathrm{~b}: k_{0} \neq 0$

The reduction formula is

$$
\begin{aligned}
& u=\left(2 h+k_{0} y\right)^{2} w(t, z)+\frac{k_{0} g^{\prime}+2\left(h+k_{0} y\right) h^{\prime \prime}}{2 k_{0}^{2}} \\
& r=t, \quad z=\frac{1}{\left(2 h+k_{0} y\right)^{2}}\left(x+\frac{k_{0} g+2\left(h+k_{0} y\right) h^{\prime}}{2 k_{0}^{2}}\right)
\end{aligned}
$$

and the corresponding reduced equation in which we may set $k_{0}=1$ is

$$
-2 k_{0}^{2} w+2 k_{0}^{2} z w_{z}-w_{z}^{2}+w_{z, t}-\left(w+4 k_{0}^{2} z^{2}\right) w_{z, z}=0 .
$$

$\underline{\text { Case } 4}: f \neq 0$

In this case the coefficient of $\partial_{t}$ in the expression of the operator $\mathbf{z}_{f}$ is nonzero and thus we may no longer consider the variable $t$ as trivially invariant. We make the simplifying assumption that in (10) we have $k_{0}=0$, so that we are looking for a reduction of the equation by a generic vector field of the form

$$
\mathbf{v}=\mathbf{x}_{g}+\mathbf{y}_{h}+\mathbf{z}_{f} .
$$

The expressions for the invariant functions $w, z$, and $r$ are found after some long calculations to be given by

$$
\begin{aligned}
r= & \frac{1}{f^{2 / 3}}\left(y-\frac{1}{3} f^{2 / 3} G\right) \\
z= & \frac{1}{54 f^{4 / 3}}\left[54 x f+3 f^{2 / 3} h G-9 y\left(h+y f^{\prime}\right)\right] \\
& +\frac{1}{54}\left[-9 \int\left(\frac{g}{f^{4 / 3}}+\frac{G h^{\prime}}{3 f^{2 / 3}}+\frac{1}{9} G^{2} f^{\prime \prime}\right) d t+G^{2} f^{\prime}\right] \\
w= & \frac{36 u f^{2}-\left(h+2 y f^{\prime}\right)^{2}+6 f\left(g+2 x f^{\prime}+y\left(h^{\prime}+y f^{\prime \prime}\right)\right)}{36 f^{4 / 3}},
\end{aligned}
$$


where

$$
G=\int \frac{h}{f^{5 / 3}} d t .
$$

The reduced equation in this case is

$$
w_{z}^{2}+w w_{z, z}+w_{r, r}=0 .
$$

It appears that equations (22) and (32) are exactly the same, despite the long calculations leading to the latter equation, and this confirms in some sense the results of part (c) of Lemma 1 and that of Lemma 2 which assert that when $k_{0}=0$, and $f \neq 0$, the corresponding vector $\mathbf{v}$ of (30) is equivalent to $\mathbf{z}_{(1)}$. In a similar way, in the case where $f \neq 0$ and $k_{0} \neq 0$, the equation obtained by direct case analysis, although not calculated in this paper, should match that obtained in (21). Similar correspondences can be established between the equations obtained in Section 4.1.1 and Section 4.1.2.

The classification obtained by direct case analysis in this section contains an exhaustive classification of all possible similarity reductions by one dimensional subgroups, except the case when $k_{0} \neq 0$ and $f \neq 0$.

4.2. Reduction by two-dimensional subgroups. Despite the list of all twelve canonical forms of non-equivalent two-dimensional subalgebras of the ZK symmetry algebra given in Section 3.2.2 we shall only consider reductions of the equation by all pairs of distinct generators of the symmetry algebra (except those pairs of the form $\left\{\mathbf{v}_{F_{1}}, \mathbf{v}_{F_{2}}\right\}$ defined by the same type of generators). This is because space limitations precludes the treatment of all twelve cases in this paper. Also, as indicated in [11, these reductions do not yield additional solutions when compared with those obtained from reductions by one-dimensional subgroups. However, they give rise to equations which are normally easier to solve. Each of the pairs of generators that we shall consider generates a subalgebra whose corresponding action turns out to have orbits of dimension two, and thus reduces the equation to an ODE. We shall always indicate to which of the classified canonical forms of twodimensional subalgebras they correspond.

The similarity variables in terms of which the reduced equations are expressed will be found by solving a system of first order partial differential equation of the form

$$
\left\{\begin{array}{l}
\mathbf{v}_{F_{1}} \cdot I=0 \\
\mathbf{w}_{F_{2}} \cdot I=0
\end{array}\right.
$$

where each $\mathbf{v}$ and $\mathbf{w}$ are operators depending on the functions $F_{1}$ and $F_{2}$, respectively. Because of the arbitrary functions appearing in (33), it can be difficult to solve this system directly in certain cases. However, Equation (33) becomes more tractable when it is transformed into the equivalent adjoint system of total differential equation. The process for this transformation and the method for solving the resulting system of total differential equations are described in [20, 21].

One important aspect of Equation (33) is its integrability condition. If we consider for example this system with $\mathbf{v}_{F_{1}}=\mathbf{x}_{g}$, and $\mathbf{w}_{F_{2}}=\mathbf{z}_{f}$ (see Equation (17)) and $f(t)=1$, then we have $\mathbf{x}_{g} \equiv(0, g, 0,-g)$ and $\mathbf{z}_{f} \equiv(1,0,0,0)$. We may thus ignore the variable $y$, in which case a function $F(x, u)$ is an invariant if and only if 
$\mathbf{x}_{g} \cdot F(x, u)=0$. This in turn is equivalent to

$$
\frac{g^{\prime}}{g}=\frac{F_{x}(x, u)}{F_{u}(x, u)}=a, \quad \text { where } a \text { is a constant. }
$$

This shows that the function $g$ of the operator $\mathbf{x}_{g}$ most satisfy $g=C e^{a t}$, for some constant $C$. More generally, for an arbitrary pair $\{g, f\}$, the compatibility condition for Equation (33) is given by

$$
-3 f g^{2}-g^{2} f^{\prime \prime}+3 g\left(f^{\prime} g^{\prime}+f g^{\prime \prime}\right)=0
$$

This means that for any solution of (33) to exist, we ought to have in this case

$$
g^{3}=f \exp \left(\int \frac{K}{f} d t\right) .
$$

When the integrability condition is satisfied, Equation (33) will yield two invariants $w$, and $z$, and in order to obtain the reduced equation we shall set $w=w(z)$.

Reduction by $\left\{\mathbf{v}_{0}, \mathbf{x}_{g}\right\}$

The corresponding Lie algebra belongs to type $\mathcal{L}_{2,9}$. The reduction formula is

$$
u=g^{\prime} y^{2}\left(w(z)-\frac{x}{g y^{2}}\right), \quad z=t
$$

and the reduced equation is

$$
-2 w g^{\prime}-\frac{g^{\prime \prime}}{g}=0
$$

This yields the group-invariant solution

$$
u=-y^{2} g^{\prime}\left(\frac{x}{y^{2} g}+\frac{g^{\prime \prime}}{2 g g^{\prime}}\right) .
$$

\section{Reduction by $\left\{\mathbf{v}_{0}, \mathbf{y}_{h}\right\}$}

This Lie algebra belongs to the family of type $\mathcal{L}_{2,11}$ Lie algebras. The reduction formula is

$$
u=\left[\left(\frac{2 x}{h^{\prime}}-\frac{y^{2}}{2 h}\right) w(z)-\frac{y^{2}}{2 h}\right] \frac{h^{\prime \prime}}{2}, \quad z=t .
$$

The reduced equation is a generalized Riccati equation of the form

$$
w^{\prime}+\alpha(t) w^{2}+\beta(t) w+\gamma(t)=0
$$

where $\alpha(t), \beta(t)$, and $\gamma(t)$ are some functions of time.

Reduction by $\left\{\mathbf{x}_{g}, \mathbf{y}_{h}\right\}$

This is a Lie algebra of type $\mathcal{L}_{2,4}$. The reduction formula is

$$
u=\frac{1}{2 h g}\left[w(z)-2 h g^{\prime} x+\left(g^{\prime} h^{\prime}-g h^{\prime \prime}\right) \frac{y^{2}}{2}\right], \quad z=t .
$$

The reduced equation corresponds in this case to a mere integrability condition of the form

$$
-g^{\prime} h^{\prime}+2 h g^{\prime \prime}-g h^{\prime \prime}=0 .
$$


A first integral for this last equation is

$$
h^{\prime}-2 \frac{g^{\prime}}{g} h+\frac{K}{g}=0, \quad K=\text { constant }
$$

and this leads to a functional relation between $h$ and $g$ of the form

$$
h=\alpha g^{2}-K g^{2} \int g^{-3} d t, \quad \alpha \equiv \text { constant. }
$$

The corresponding exact solution of (10) is thus

$$
u=\frac{w(t)-g^{2}\left(2 x g^{\prime}+y^{2} g^{\prime \prime}\right)\left(\alpha-K \int g^{-3} d t\right)}{2 g^{3}\left(\alpha-K \int g^{-3} d t\right)}
$$

where $w$ is an arbitrary function of time. For $K=0$, it reduces to

$$
u=\frac{w(t)-\alpha g^{2}\left(2 x g^{\prime}+y^{2} g^{\prime \prime}\right)}{2 \alpha g^{3}} .
$$

Reduction by $\left\{\mathbf{v}_{0}, \mathbf{z}_{f}\right\}$

This is a particular case of type $\mathcal{L}_{2,1}$ Lie algebras. The equivalent system of total differential equations takes in this case the form

$$
\begin{aligned}
& d y=\frac{6 x y f^{\prime}-y^{3} f^{\prime \prime}}{12 x f} d t+\frac{y}{2 x} d x \\
& d u=\frac{1}{12 x f}\left[-12 u x f^{\prime}-4 x^{2} f^{\prime \prime}-2 u y^{2} f^{\prime \prime}-2 x y^{2} f^{(3)}\right] d t+\frac{u}{x} d x .
\end{aligned}
$$

This leads to the reduction formula

$$
\begin{aligned}
& u=\frac{y^{2}}{6 f^{2}}\left[w(z)+\frac{2}{3} f^{\prime 2}-\left(\frac{2 x}{y^{2}} f^{\prime}+f^{\prime \prime}\right) f\right] \\
& z=\frac{f x}{y^{2}}-\frac{f^{\prime}}{6}
\end{aligned}
$$

and the reduced equation is

$$
12 w-12 z w^{\prime}+w^{2}+\left(w+24 z^{2}\right) w^{\prime \prime}=0 .
$$

The solution for $w=0$ is

$$
u=\frac{y^{2}}{6 f^{2}}\left[\frac{2}{3} f^{\prime 2}-\left(\frac{2 x}{y^{2}} f^{\prime}+f^{\prime \prime}\right) f\right] .
$$

Reduction by $\left\{\mathbf{x}_{g}, \mathbf{z}_{f}\right\}$

This Lie algebra belongs to type $\mathcal{L}_{2,10}$. The equivalent system is

$$
\begin{aligned}
& d y=\frac{2 y f^{\prime}}{3 f} d t \\
& d u=\frac{1}{6 f g}\left[-4 u g f^{\prime}+2 x f^{\prime} g^{\prime}-2 x g f^{\prime \prime}+y^{2} g^{\prime} f^{\prime \prime}-y^{2} g f^{(3)}\right] d t-\frac{g^{\prime}}{g} d x
\end{aligned}
$$

The integrability condition is

$$
-3 f g^{\prime 2}-g^{2} f^{\prime \prime}+3 g\left(f^{\prime} g^{\prime}+f g^{\prime \prime}\right)=0 .
$$

Integrating this last equation once yields

$$
3 f \frac{g^{\prime}}{g}-f^{\prime}=K \equiv \text { constant }
$$


We must therefore have

$$
g^{3}=f \exp \left(\int \frac{K}{f} d t\right)
$$

as we already indicated earlier in (34)

The reduction formula is

$$
\begin{aligned}
u & =\frac{1}{f^{2 / 3}}\left[w(z)-\frac{x\left(K+f^{\prime}\right)}{3 f^{1 / 3}}-\frac{y^{2}}{6 f^{4 / 3}}\left(-\frac{K}{3} f^{\prime}-\frac{2}{3} f^{\prime 2}+f f^{\prime \prime}\right)\right] \\
z & =y^{3} / f^{2}
\end{aligned}
$$

and the reduced equation is the linear equation

$$
9 z^{4 / 3} w^{\prime \prime}+6 z^{1 / 3} w^{\prime}+\frac{K^{2}}{9}=0
$$

with solution

$$
w(z)=-\frac{1}{18} K^{2} z^{2 / 3}+3 z^{1 / 3} a_{1}+a_{2}
$$

for some constants of integration $a_{1}$ and $a_{2}$. Substituting this expression for $w(z)$ in the reduction formula yields

$$
\begin{aligned}
u & =\frac{1}{18 f^{2}}\left[\left(18 a_{2}+54 a_{1}\left(y^{3} / f^{2}\right)^{2 / 3}-K^{2}\left(y^{3} / f^{2}\right)^{2 / 3}\right) f^{4 / 3}\right] \\
& +\frac{1}{18 f^{2}}\left(y^{2} f^{\prime}\left(K+2 f^{\prime}\right)-3 f\left(2 K x+2 x f^{\prime}+y^{2} f^{\prime \prime}\right)\right) .
\end{aligned}
$$

\section{Reduction by $\left\{\mathbf{y}_{h}, \mathbf{z}_{f}\right\}$}

This Lie algebra is of type $\mathcal{L}_{2,12}$. The equivalent system is

$$
\begin{aligned}
& d y=\left[\frac{2 y f^{\prime}}{3 f}-\frac{h\left(2 x f^{\prime}+y^{2} f^{\prime \prime}\right)}{3 y f h^{\prime}}\right] d t+\frac{2 h}{y h^{\prime}} d x \\
& d u=\left[\frac{h^{\prime \prime}\left(2 x f^{\prime}+y^{2} f^{\prime \prime}\right)}{6 f h^{\prime}}+\frac{\left(-4 u f^{\prime}-2 x f^{\prime \prime}-y^{2} f^{(3)}\right)}{6 f}\right] d t-\frac{h^{\prime \prime}}{h^{\prime}} d x
\end{aligned}
$$

and the integrability condition is given by

$$
3 f h^{\prime 2}+2 h^{2} f^{\prime \prime}-3 h\left(f^{\prime} h^{\prime}+f h^{\prime \prime}\right)=0 .
$$

Integrating this equation once gives

$$
\frac{2 f^{\prime}-K}{3 f}=\frac{h^{\prime}}{h}
$$

where $K$ is a constant of integration. Thus we must have

$$
h=C\left[f^{2} \exp \left(-\int \frac{K}{f} d t\right)\right]^{1 / 3} .
$$

The reduction formula is

$$
\begin{aligned}
& u=\frac{1}{f^{2 / 3}}\left[w(z)-\frac{y^{2} f^{\prime}\left(K-2 f^{\prime}\right)}{18 f^{4 / 3}}-\frac{-6 K x+6 x f^{\prime}+3 y^{2} f^{\prime \prime}}{18 f^{1 / 3}}\right] \\
& z=\frac{12 x f+y^{2}\left(K-2 f^{\prime}\right)}{f^{4 / 3}} .
\end{aligned}
$$

These yield the reduced equation

$$
K^{2}+90 K w^{\prime}+1296 w^{\prime 2}+36(36 w+K z) w^{\prime \prime}=0 .
$$


For $K=0$, this equation reduces to

$$
w^{\prime 2}+w w^{\prime \prime}=0
$$

with solution

$$
w=\beta \sqrt{2 z-\alpha}, \quad(\alpha \equiv \text { constant }, \quad \beta \equiv \text { constant }) .
$$

The corresponding solution of (10) is

$$
u=\frac{1}{18 f^{2}}\left[2 y^{2} f^{\prime 2}-3 f\left(2 x f^{\prime}+y^{2} f^{\prime \prime}\right)+18 \beta f^{4 / 3}\left(4 \frac{6 x f-y^{2} f^{\prime}}{f^{4 / 3}}-\alpha\right)^{1 / 2}\right] .
$$

All the exact solutions to Equation (1) that we have found depend typically on zero arbitrary functions of time, and on at most two such functions and some arbitrary constants. Whenever the solution to a reduced equation was known, we were always able to readily obtain the solution to the original equation by a mere substitution into a reduction formula of the form (18). This situation is very different in the case of the direct method of Clarkson and Kruskal that we investigate in more details in the next section. Clearly, not all the group-invariant solutions are physically relevant for the sound wave propagation problem. However, they are certainly relevant for other problems modeled by the same differential equations when the boundary conditions change.

\section{Comparison with Clarkson and Kruskal's method}

By the direct reduction method of Clarkson and Kruskal [8, all similarity solutions of the ZK equation of the form

$$
u(t, x, y)=U(t, x, y, w(z)), \quad z=z(t, x, y)
$$

where $U$ is a function of the indicated variables, and $w(z)$ satisfies an ODE, may be found by substituting Equation (49) into Equation (11). It is also argued in [7] that by a result of Clarkson and Kruskal, in order to find all such solutions, it is sufficient to look for $u$ in the form

$$
u(t, x, y)=\alpha+\beta w(z)
$$

where $\alpha$ and $\beta$ are functions of $t, x$, and $y$. The latter equality is precisely our Equation (18) that gives the general form of all possible similarity solutions that we have found thus far, in terms of the similarity variables. By making use of the reduced anzatz $z_{x} \neq 0$, Zhang et al. [7] showed in this way that the most general ODE satisfied by $w(z)$ has the form

$$
w^{\prime 2}+w w^{\prime \prime}+(A z+B) w^{\prime}+2 A w=\frac{1}{3}(A z+B)^{2}
$$

and raised the question of whether there is any connection between the direct method and the Lie classical method applied in the preceding section.

One such connection could be determined by a way of mapping the reduced equation obtained by one method, to that obtained by the other method, taking into account that the direct method gives in principle the most general equation. Such a correspondence is not obvious because of the various changes of variables through which Equation (3) was obtained, except perhaps if equations are replaced by their equivalence classes under equivalence transformations. However, due to a wellknown result asserting that two equivalent differential equations have isomorphic 
symmetry groups 22, these equivalences become easier to establish. The symmetry algebra of each of the reduced oDEs found in section 4.2 has dimension at least two, except for Equation (38) whose symmetry algebra is generated by $\mathbf{v}_{1}=z \partial_{z}+2 w \partial_{w}$ alone. For $A \neq 0$, Equation (51) is mapped after the change of independent variable $y=z+B / A$ to the equation

$$
w^{\prime 2}+w w^{\prime \prime}+(A y) w^{\prime}+2 A w-\frac{1}{3}(A y)^{2}=0
$$

which has the same symmetry algebra as Equation (38). The necessary condition for an equivalence between these two equations is thus satisfied. However, we've found that no linear fractional transformation of the form

$$
H=\frac{a_{1} w+a_{2}}{a_{3} w+a_{4}}, \quad \xi=a_{5} x+a_{6},
$$

where $a_{1}, \ldots, a_{6}$ are arbitrary constants with $a_{1} a_{4}-a_{2} a_{3} \neq 0$, maps Equation (52) to Equation (38). This does not necessarily precludes the two equations from being equivalent under other types of transformations.

For $A=0$, Equation (51) reduces to

$$
-\frac{B^{2}}{3}+B w^{\prime}+w^{\prime 2}+w w^{\prime \prime}=0
$$

and the corresponding symmetry algebra has generators $\mathbf{v}_{1}=\partial_{z}, \mathbf{v}_{2}=z \partial_{z}+w \partial_{w}$. This symmetry algebra has the same dimension as that for the reduced Equation (45) from section 4.2, Equation (45) with $K=0$ is exactly the same as Equation (53) with $B=0$. For $K \neq 0$, the change of variables

$$
v=K m(36 w+k z), \quad y=-K z / 36, \quad \text { with } m=\frac{-35+3 \sqrt{21}}{36288}
$$

reduces Equation (45) to an equation of the form

$$
-\frac{B^{2}}{3}+B v^{\prime}+v^{\prime 2}+v v^{\prime \prime}=0
$$

with $B=(-9+5 \sqrt{21}) K / 672$. This shows the equivalence of the equations (53) and (45).

We thus see that by using the symmetry properties of the reduced equations, we can always map each of the symmetry-reduced equations to a sub-equation of (51). It should be noted that equations obtained by the direct method of Clarkson and Kruskal tend to be broad in nature and hence more difficult to solve. In addition, no symmetry or other properties of the solutions these equations are known, and there is also no guarantee that the solutions to the original equation is recoverable from those of the reduced equation, because the process of reverting back the solution generally involves solving other PDEs. When this process does give rise to an explicit solution of the original equation, it usually involves relatively complicated expressions. For example, the full expression of the exact solution generated by the reduced Equation (46) and given by (47) is easily found in Lie's approach and depends only on one arbitrary function of time and two arbitrary constants. The same solution is obtained by the direct method in [7] only through the solving a number of intermediary PDEs, and depends on no less than four arbitrary functions of time plus the two arbitrary constants.

As for the reduction to $(1+1)$-dimensional equations, the similarity solutions and corresponding reduced equations found in Section 4.1 are much simpler and 
complete than those obtained using the direct method of Clarkson and Kruskal in 7], where both the equations and corresponding solutions depend on up to four arbitrary functions and are also usually defined only implicitly in terms of the solutions of some PDEs. For instance, if a general reduced equation of the form (32) were to be determined by the direct method, it would more likely be determined only implicitly and be expressed in terms of unnecessary arbitrary functions. Although the direct method also gives in principle the similarity solution as well as the reduced equation, its algorithm is relatively complicated and yields most often complicated and incomplete results.

In the classical Lie reduction approach, the symmetry properties of each similarity solution is known and in some cases, especially in the case of solvable symmetry algebras, the solution to the original equation can be recovered by quadratures.

It should however also be noted that even with Lie's classical method, having to revert back to the solution of the original equation by quadratures alone does not guarantee the explicit determination of this solution. Indeed, these quadratures may involve transcendental or integral equations that aren't easy to solve. This fact is common in the determination of group-invariant solutions and we give another example here by attempting to solve Equation (45), for which the symmetry generators are given by

$$
\mathbf{v}_{1}=(-36 / k) \partial_{z}+\partial_{w}, \quad \text { and } \quad \mathbf{v}_{2}=z \partial_{z}+w \partial_{w} .
$$

These vector fields clearly generate a solvable Lie algebra with commutation relations $\left[\mathbf{v}_{1}, \mathbf{v}_{2}\right]=\mathbf{v}_{1}$. In terms of the rectifying coordinates

$$
y=36 w+K z \quad \text { and } r=-(K x) / 36
$$

for $\mathbf{v}_{1}$, Equation (45) reduces to

where

$$
\begin{aligned}
-H+18 H^{2}+648 H^{3}+y H^{\prime} & =0 \\
H & =\frac{d r}{d y} .
\end{aligned}
$$

Equation (55) retains the symmetry $\mathbf{v}_{2}$, which is given by $\tilde{\mathbf{v}}_{2}=y \partial_{y}+r \partial_{r}$ in terms of the variables $r$ and $y$, and by

$$
\hat{\mathbf{v}}_{2}=\frac{H}{(1+18 H)^{1 / 3}(36 H-1)^{2 / 3}} \partial_{y}
$$

in terms of $H$ and $y$. In terms of the rectifying coordinates

$$
\begin{aligned}
& \xi=H \\
& S=\frac{(1+18 H)^{1 / 3}(36 H-1)^{2 / 3}}{H} y
\end{aligned}
$$

for $\hat{\mathbf{v}}_{2}$, Equation (55) reduces to

$$
\xi^{3}(1+18 \xi)^{5 / 3}(36 \xi-1)^{4 / 3} S^{\prime}=0
$$

with solution $S(\xi)=a$, where $a$ is a constant of integration. It thus follows from Equation (59) that the solution $H=F(y)$ of (55) is given implicitly by the polynomial equation

$$
a H^{3}-y^{3}+54 H y^{3}-23328 H^{3} y^{3}=0 .
$$

Assuming that the function $F(y)$ is known, it readily follows from Equation (56) that 


$$
r=\int F(y) d y=G(y)
$$

for a certain function $G(y)$, and the substitution of (54) and (44) into this last equation leads to the solution to the original equation by an additional quadrature. The problem here with these quadratures is that the integral $r=\int F(y) d y$ is not easy to solve because of the complicated form of the function $F$. Indeed, one of the simplest roots $H=F(y)$ of the polynomial Equation (60) is given by

$$
F(y)=\frac{-36 a y^{3}+839808 y^{6}+2^{1 / 3}\left(y^{3} X^{2}+\sqrt{a y^{6} X^{3}}\right)^{2 / 3}}{2^{2 / 3} X\left(y^{3} X^{2}+\sqrt{a y^{6} X^{3}}\right)^{1 / 3}}
$$

where $X=a-23328 y^{3}$, and so the integral equation $r=\int F(y) d y$ does not appear to be obvious to solve, unless perhaps an appropriate change of variable to be found was performed. In reality, the difficulties with these quadratures are normally to be associated with the equation itself and not with Lie's reduction method.

\section{Concluding Remarks}

In this paper we have given a classification of low-dimensional subalgebras of the ZK symmetry algebra into conjugacy classes and determined similarity reductions of the ZK equation to $(1+1)$-dimensional equations and to ODEs. We have thus derived a number of new exact solutions to this equation in this way. Consequently, we have been able to compare the results obtained using Lie's classical method with those obtained in [7] with the direct method of Clarkson and Kruskal, and found in particular equivalence transformations between the reduced equations obtained by this two methods. This investigation shows that not only Lie's algorithm is simpler and much richer in properties, but it also yields simpler and more complete results as opposed to the direct method where both solutions and reduced equations are most often determined only implicitly by complicated expressions.

The example of reduction by direct case analysis treated in Section 4.1 .2 suggests that equations obtained by the direct method can also be viewed as reduced equations obtained by the Lie classical method by performing a reduction with a system of generic generators corresponding to Lie algebras having orbits of fixed dimensions, rather than performing the reduction with some specific generators.

\section{REFERENCES}

[1] Zabolotskaya, E.A., and Khokhlov, R.V.: Quasi plane waves in the nonlinear acoustics of confined beams. Soviet Phys. Acoust. 15, 35-40 (1969)

[2] Sarvazyan, A.P., Rudenko, O.V., Swanson, S.D., Fowlkes, J.B., and Emelianov, S.Y.: Shear wave elasticity imaging: A new ultrasonic technology of medical diagnostics. Ultrasound Med. Biol. 24, 1419-1435 (1999)

[3] Bakhvalov, N.S., Zhileikin, Y.M., and Zabolotskaya, E.A.: Nonlinear Theory of Sound Beams. Amer. Inst. of Phys, New York (1987)

[4] Chowdhury, A.R., and Nasker, M.: Towards the conservation laws and Lie symmetries for the Khokhlov-Zabolotskaya equation in three dimensions. J. Phys. A: Math. Gen. 19, 1775-1782 (1986)

[5] Schwarz, F.: Symmetries of the Khokhlov-Zabolotskaya equation. J. Phys. A: Math. Gen. 20, 1613-1614 (1987)

[6] Hereman, W., Steeb, W.H., and Euler N.: Towards the conservation laws and Lie symmetries for the Khokhlov-Zabolotskaya equation in three dimensions. J. Phys. A: Math. Gen. 25, 2417-2418 (1992) 
[7] Zhang, J.F., Zhu, Y.J., and Lin, J.: Similarity reductions of the Khokhlov-Zabolotskaya Equation. Commun. Theor. Phys. 24, 69-74 (1995)

[8] Clarkson, P.A., and Kruskal, M.D.: New Similarity reductions of the Boussinesq Equation. J. Math. Phys. 30, 2201-2213 (1989)

[9] Olver, P.J.: Applications of Lie Groups to Differential Equations. Springer, New York (1986)

[10] Stephani, H.: Differential Equations. Cambridge University Press, Cambridge (1989)

[11] David, D., Kamran, N., Levi, D., and Winternitz, P.: Symmetry reduction for the KadomtsevPetviashvili equation using a loop algebra. J. Math. Phys. 27, 1225-1237 (1986)

[12] Cartan, E.: Oeuvres Complètes. CNRS, Paris (1984)

[13] Winternitz, P.: Kac-Moody-Virasoro symmetries of integrable nonlinear partial differential equations. Preprint CRM 1548 (1988)

[14] Gungor, F.: On the Virassoro structure of symmetry algebras of nonlinear partial differential equations. Symmetry, Integrability, and Geometry: Methods and Applications. 2, 014 (2006)

[15] Sentil, Velan M., and Lakshmanan, M.: Kac-Moody-Virasoro algebras and integrability of certain (2+1)-dimensional nonlinear evolution equations. J. Nonlinear Math. Phys. 5, 190-211 (1998)

[16] Ovsyannikov, L. V. Group analysis of Differential Equations. Nauka, Moscow (1978)

[17] Winternitz, P.: Lie groups and solutions of nonlinear partial differential equations. Preprint CRM-1841 (1993)

[18] Gibbons, J., and Kodama, Y.: Integrable quasilinear systems: generalized hodograph transformation Nonlinear evolutions (Teaneck: World Sci. Publ.) (1987)

[19] Neuman, F.: ?? 23rd International Symposium on Functional Equations (Italy: Gragnano) (?).

[20] Ndogmo, J.C.: Invariants of a semi-direct sum of Lie algebras. J. Phys. A: Math. Gen. 37, 5635-5647 (2004)

[21] Forsyth, A.R.: Theory of Differential Equations. Part 1. Dover, New York (1890)

[22] Olver, P.J.: Equivalence, Invariants, and Symmetry. Cambridge University Press, Cambridge (1995)

P O Box 2446, Bellville 7535, South AfricA.

E-mail address: jndogmo@uwc.ac.za 